\title{
Effective Capacity and Interference Analysis in Multiband Dynamic Spectrum Sensing
}

\author{
Mohamed Elalem, Lian Zhao \\ Electrical and Computer Engineering Department, Ryerson University, Toronto, Canada \\ Email: melalem@ryerson.ca, lzhao@ee.ryerson.ca
}

Received February 11, 2013; revised March 11, 2013; accepted April 11, 2013

Copyright (c) 2013 Mohamed Elalem, Lian Zhao. This is an open access article distributed under the Creative Commons Attribution License, which permits unrestricted use, distribution, and reproduction in any medium, provided the original work is properly cited.

\begin{abstract}
In this paper, the performance of multichannel transmission in cognitive radio is studied. Both $Q o S$ constraints and interference limitations are considered. The activities of the primary users (PU)s are initially detected by cognitive users (CU)s who perform sensing process over multiple channels. They transmit in a single channel at variable power and rates depending on the channel sensing decisions and the fading environment. The cognitive operation is modeled as a state transition model in which all possible scenarios are studied. The $Q o S$ constraint of the cognitive users is investigated through statistical analysis. Analytical form for the effective capacity of the cognitive radio channel is found. Optimal power allocation and optimal channel selection criterion are obtained. Impact of several parameters on the transmission performance, as channel sensing parameters, number of available channels, fading and other, are identified through numerical example.
\end{abstract}

Keywords: Effective Capacity; Cognitive Radio; Multiband Sensing; Interference

\section{Introduction}

Much interest in cognitive radio systems is raised due to their ability to utilize the available spectrum much more effectively. In addition to efficient spectrum utilization, it is more important in wireless systems to provide reliable communications while preserving a certain level of quality-of-service $(Q o S)$. In cognitive radio systems, challenges in providing $Q o S$ assurances increase due to the fact that cognitive users should operate under constraints on the interference levels that they produce to primary users. For the secondary users, these interference constraints lead to variations in transmit power levels and channel accesses. This discontinuing in accessing the channels due to the activity of primary users make it difficult for the cognitive users to satisfy their own $Q o S$ requirement. The authors in [1] proposed a $Q o S$ constrained power and rate allocation scheme for spectrum sharing systems in which it was guaranteed a minimumrate to the primary user for a certain percentage of time. The same authors, in [2], considered variable-rate variable-power modulation employed under delay $Q o S$ constraints over spectrum-sharing channels. Various capacity metrics, e.g., ergodic, outage, and minimum-rate capacity for the cognitive channels with interference constraints in Rayleigh fading environments, have been stud- ied in these papers.

In this paper, the effective capacity of cognitive radio channels is studied where the cognitive radio detects the activity of the primary users in a multichannel case, and it performs data transmission in one of these channels using both rate and power adaptation that depends on the channel conditions and the activity of the primary users. An average interference constraint on the cognitive users is formulated. Maximum throughput formula is obtained through the effective capacity approach. Optimal power allocation is derived and investigated through numerical results.

The rest of the paper is organized as follows. In Section 2, the system model and assumptions are given. Channel capacity and state transition model are formulated and constructed in Section 3. Section 4 discusses outage constraints and interference limit. In Section 5, the effective capacity for the cognitive user is formally defined in terms of $Q o S$ constraints and then formulated and optimized. Channel selection criterion is proposed in Section 6. Numerical results are provided in Section 7, and the paper is concluded in Section 8.

\section{System Model and Assumptions}

The considered system model consists of cognitive radio 
network in which the cognitive users sense $M$ channels with a bandwidth $B_{m}$ for each, where $m=1,2, \cdots, M$ among them the cognitive users choose a channel for data transmission. The channel sensing and data transmission are performed in frames of duration $T$. It is assumed that $\tau$ seconds is allocated for channel sensing while the remaining $(T-\tau)$ seconds are allocated for data transmission. The primary user activity influences the transmission power and rate levels. If all of the channels are detected as busy, the cognitive transmitter selects one channel according to a certain criterion, and sets the transmission power and rate to $S_{b, m}(i)$ and $r_{b, m}(i)$, where $i=1,2, \cdots$ denotes the time index in the transmission frame. In the contrary, if at least one channel is sensed to be idle, data transmission is sent with power $S_{d, m}(i)$ at rate $r_{d, m}(i)$. In the case of multiple channels are detected as idle, one idle channel is selected according to a certain policy explained later.

The channel fading coefficient between the cognitive transmitter and the receiver with an arbitrary distribution is assumed as $g_{m}(i)$. A block-fading channel model is assumed in which the fading coefficients stay constant in the frame, and they may change from one block to another independently in each channel. The active primary user's signal arriving at the cognitive receiver is assumed to have zero mean and a variance $\sigma_{\zeta_{m}}^{2}$. The background noise at the receivers is modeled as a zero-mean, circularly symmetric, complex Gaussian random variable with variance $\sigma_{n_{m}}^{2}$.

Perfect Channel State Information (CSI) is assumed at each receiver. It is further assumed that each channel has a bandwidth that is equal to the coherence bandwidth $B_{c}$. With this assumption, it can be supposed that independent flat fading is experienced in each channel.

\section{Channel Capacity and State Transition Model}

In order to further simplify the setting, it is considered a symmetric model in which fading coefficients are identically distributed in different channels. Moreover, the background noise and primary users' signals are also assumed to be identically distributed in different channels and hence their variances $\sigma_{n}^{2}$ and $\sigma_{\zeta}^{2}$ do not depend on $m$. So the subscript $m$ can be omitted. The probability of each channel being occupied by the primary users is assumed to be the same and equal to $q$. In channel sensing process, the same energy threshold is assumed in each channel.

Due to possible error in channel sensing, we may have the following possible cases:

- $C_{b b}: M$ bands are sensed as busy, and the selected channel is actually busy.

- $C_{b d}: M$ bands are sensed as busy, and the selected channel is actually idle.
- $C_{d b}: M_{d}$ band(s) is sensed as idle, and the selected channel is actually busy.

- $C_{d d}: M_{d}$ band(s) is sensed as idle, and the selected channel is actually idle, where $M_{d}=1, \cdots, M-1$.

By the assumption of that the state transitions occur every frame, the probability of the channel detected as busy can be written as

$$
\rho \triangleq\left(q p_{d}+(1-q) p_{f}\right),
$$

where $p_{d}$, and $p_{f}$ are the probabilities of detection and false alarm. The probabilities of being in each of the above cases are summarized in Table 1.

In each of the above cases, we have two states, namely either ON state in which the instantaneous transmission rate exceeds the instantaneous channel capacity, or OFF state if it does not. Assuming the interference caused by the primary users $\zeta(i)$ as additional Gaussian noise, the instantaneous channel capacities for the above four cases can be expressed as follows

$$
R_{l}(i)=B_{c} \log _{2}\left(1+\gamma_{l}(i)\right),
$$

where $l \in\{b b, b d, d b, d d\}$ refers to the possible case and $\gamma_{l}(i)$ represents the SNIR for the corresponding case in the $i^{\text {th }}$ channel which is given by

$$
\begin{aligned}
& \gamma_{b b}(i)=\frac{S_{b}(i) g(i)}{N_{b}}, \gamma_{b d}(i)=\frac{S_{b}(i) g(i)}{N_{d}} \\
& \gamma_{d b}(i)=\frac{S_{d}(i) g(i)}{N_{b}}, \gamma_{d d}(i)=\frac{S_{d}(i) g(i)}{N_{d}},
\end{aligned}
$$

where $N_{b}=\left(\sigma_{n}^{2}+\sigma_{\zeta}^{2}\right)$ and $N_{d}=\sigma_{n}^{2}$ represent the total noise when a channel is busy and idle respectively.

For the cases $C_{b b}$ and $C_{b d}$, the cognitive transmitter detects all channels as busy and transmits with rate

$$
r_{b}(i)=B_{c} \log _{2}\left(1+\gamma_{b b}(i)\right) .
$$

While for the cases $C_{d b}$ and $C_{d d}$, at least one channel is sensed as idle and the transmission rate is

$$
r_{d}(i)=B_{c} \log _{2}\left(1+\gamma_{d d}(i)\right),
$$

whereas the transmitter assumes the channel as idle, it

Table 1. The probability of being in each four cases.

\begin{tabular}{cc}
\hline Case & Probability being in this case \\
\hline$C_{b b}$ & $\rho^{M-1} q p_{d}$ \\
$C_{b d}$ & $\rho^{M-1}(1-q) p_{f}$ \\
$C_{d b}$ & $\frac{\left(1-\rho^{M}\right) q\left(1-p_{d}\right)}{1-\rho}$ \\
$C_{d d}$ & $\frac{\left(1-\rho^{M}\right)(1-q)\left(1-p_{f}\right)}{1-\rho}$ \\
\hline
\end{tabular}


sets the power level to $S_{d}(i)$ and it supposes that no interference from the primary transmissions will be produced as seen by nonexistence of $\sigma_{\zeta}^{2}$ in the denominator of $\gamma_{d d}$.

The transmission rate for the cases $C_{b b}$ and $C_{b d}$ is less than or equal to the instantaneous channel capacity. Reliable transmission at rate $r_{b}(i)$ is achieved and channel is in the ON state. Similarly, the channel is in the ON state in the case $C_{d d}$ in which the transmission rate is $r_{d}(i)$. While in the case $C_{d b}$, the channel is in the OFF state because the transmission rate exceeds the instantaneous channel capacity (i.e., $\left.r_{d}(i)>R_{d b}(i)\right)$. Hence, reliable communication cannot be established.

Referring to the above discussion, the cognitive transmission model can constructed as depicted in Figure 1. We have $(M+2)$ states model, consisting of $(M+1)$ ON states and one OFF state. The first ON state represents the ON states in the cases $C_{b b}$ and $C_{b d}$, in both of which the transmission power and rate are $S_{b}(i)$ and $r_{b}(i)$ respectively. All channels are sensed as busy in this state. The remaining ON states from 2 to $(M+1)$ can be represented as the expansion of the $\mathrm{ON}$ state in the cases $C_{d d}$ in which at least one channel is sensed as idle and the selected channel for transmission is really idle. More specifically, the $m^{\text {th }}$ ON state for $m=2,3, \cdots,(M+1)$ is the ON state in which $(m-1)$ channels are sensed as idle and the channel selected for transmission is actually idle. The transmission power and rate for these states are $S_{d}(i)$ and $r_{d}(i)$ respectively.

The single $(M+2)$ OFF state represents the case $C_{d b}$ in which transmission rate exceeds the instantaneous channel capacity.

The state transition probabilities can be analyzed as-

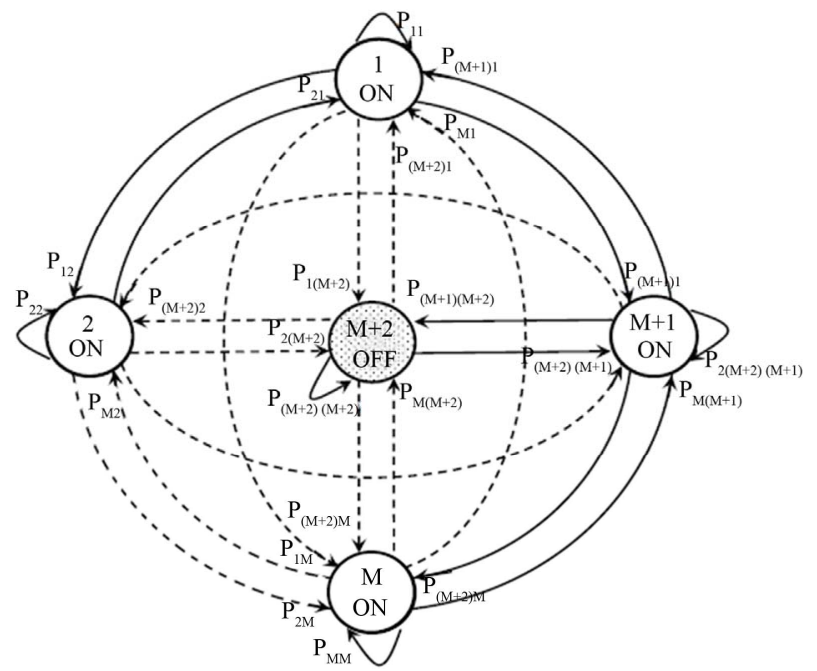

Figure 1. $(M+2)$ ON states and one OFF state for the cognitive radio channel and their corresponding state transition probabilities. suming that the PU's activity is independent among the channels and also from one frame to another as mentioned above. The probability of staying in the first $\mathrm{ON}$ state in which all channels are sensed as busy can be easily expressed as $p_{11}=\rho^{M} \triangleq p_{1}$. Again with this assumption of independence over the frames, the state transition probabilities are independent on the originating state, so the transition probabilities $p_{m 1}, m=1,2, \cdots,(M+2)$ are similar and equal to $p_{1}$. For $m=2,3, \cdots, M+1$, the transition probabilities are obtained as

$$
\begin{aligned}
& p_{m} \triangleq p_{1 m}=p_{2 m}=\cdots=p_{(M+1) m}=p_{(M+2) m} \\
& =\operatorname{Pr}\{(m-1) \text { out of } M \text { chs are sensed idle }\} \\
& \quad \times \operatorname{Pr}\{\text { selected ch is idle it is sensed as idle }\} \\
& =\left(\begin{array}{c}
M \\
m-1
\end{array}\right) \rho^{M-m+1}(1-\rho)^{m-1} \times \frac{(1-q)\left(1-p_{f}\right)}{1-\rho} \\
& =C(M, m-1) \rho^{M-m+1}(1-\rho)^{m-2}(1-q)\left(1-p_{f}\right)
\end{aligned}
$$

where $C(k, l)=\frac{k !}{l !(k-l) !}$ is the Binomial coefficient.

The transition probabilities for the OFF state are

$$
\begin{aligned}
& p_{M+2} \triangleq p_{1(M+2)}=p_{2(M+2)}=\cdots=p_{(M+1)(M+2)} \\
& =p_{(M+2)(M+2)}=1-\sum_{m=1}^{M+1} p_{1 m} \\
& =\sum_{m=1}^{M} C(M, m) \rho^{M-m}(1-\rho)^{m-1} q\left(1-p_{d}\right) \\
& =\frac{1-\rho^{M}}{1-\rho} q\left(1-p_{d}\right),
\end{aligned}
$$

where we use the power series relation in [3,4].

$$
\sum_{m=1}^{M} \frac{M !}{(M-m) ! m !} a^{M-m}(1-a)^{m-1}=\frac{1-a^{M}}{1-a}
$$

From the above definitions, it can be seen that the state transition probability matrix is $(M+2) \times(M+2)$ with a rank of 1 .

\section{Outage Constraints and Interference Limit}

To adapt the transmission powers of the CRs and ensuring not producing any harmful on the primary users, interference power constraints is analyzed in this section. From the considered system model, it can be included that the interference to the primary users is caused only in the cases $C_{b b}$ and $C_{d b}$. Where, in the case $C_{b b}$, the channel is actually busy, and the cognitive user, detecting the channel as busy, transmits at power level $S_{b}$. The instantaneous interference power introduced on the primary user is $S_{b} g_{c p}$, where $g_{c p}$ is the fading coefficient 
of the channel between the cognitive transmitter and the primary user.

For the case $C_{d b}$, although the channel is actually busy, the secondary user, detecting the channel as idle, transmits at power $S_{d}$. The instantaneous interference power is $S_{d} g_{c p}$ in this case. Since power adaption is considered, transmission power levels $S_{b}$ and $S_{d}$ in general vary with $g_{c p}$ and $g$, which is the power of the fading coefficient between the secondary transmitter and secondary receiver in the chosen transmission channel.

In the cases: $C_{b b}$ and $C_{d b}$, the instantaneous interfer- ence power levels depend on both $g_{c p}$ and $g$ whose distributions depend on number of available channels from which the selection is performed. For this reason, it is necessary in the case $C_{d b}$ to separately consider the individual cases with different number of idle-detected channels. For instance, in the $m^{\text {th }}$ case for

$m=1,2, \cdots, M$, there are $m$ channels detected as idle and the channel chosen out of these $m$ channels is actually busy.

The average interference has to be constrained to the value $I_{m}^{\text {th }}$. This can be formulated as

$$
I_{m}^{\text {th }}>\operatorname{Pr}\left\{\text { being in } C_{b b}\right\} \times \text { average interf. in } C_{b b}+\operatorname{Pr}\left\{\text { being in } C_{d b}\right\} \times \text { average interf. in } C_{d b} \text {. }
$$

This yields to

$$
I_{m}^{t h}>\left[\rho^{M-1} q p_{d} \times \mathbb{E}\left[S_{b} g_{c p}\right]+\frac{\left(1-\rho^{M}\right)(1-q)\left(1-p_{f}\right)}{1-\rho} \times \mathbb{E}_{m}\left[S_{d} g_{c p}\right]\right]
$$

The expectations should be taken over the distributions of $g$ and $g_{c p}$ and over the probabilities of different cases. The term $\mathbb{E}_{m}\left[S_{d} g_{c p}\right]$ in Equation (9) depends on the number of idle-detected channels, $m$.

\section{Effective Capacity for Cognitive User}

The Effective Capacity $\left(E_{C}\right)$ (or Effective Bandwidth) theory is a powerful approach to evaluate the capability of a wireless channel to support data transmissions with diverse statistical quality of service $Q o S$ guarantees [5-7]. It is defined as the maximum constant arrival rate that can be supported by a given channel service process while meeting the $Q o S$ requirement [5]. In particular, the statistical $Q o S$ guarantee can be characterized by a metric called $Q \circ S$ exponent denoted by $\theta, 0<\theta<\infty$ [5]. The $Q o S$ exponent $\theta$ characterizes the exponentially decaying rate of the violation probability against the queue-length threshold. With the pair (Effective Capacity $E_{C}$ and $Q o S$ exponent $\theta$ ), it can be observed insight tradeoff between the delay $Q o S$ requirement and the system throughput. Higher $\theta$ represents more stringent delay $Q o S$ requirements, and vice versa.

The effective capacity for a given $\theta$ is defined in [5] as

$$
\frac{\Lambda(-\theta)}{\theta}=-\lim _{t \rightarrow \infty} \frac{1}{\theta t} \log \mathbb{E}(\exp (-\theta R(t)))
$$

where $\Lambda(\theta)=\lim _{t \rightarrow \infty} \frac{1}{t} \log \mathbb{E}(\exp (-\theta R(t)))$ is a function that depends on the natural logarithm of the moment generating function of $R(t)$, and $R(t)=\sum_{i=1}^{t} r(i)$ is the time-accumulated service process. $\mathbb{E}(\cdot)$ is the expectation with respect to $r$.

It can be noticed that the service rate is $r(m)=r_{b}(m)(T-\tau)$ if the cognitive user is in state 1 at band $m$. Similarly, the service rate is $r(m)=r_{d}(T-\tau)$ in states between 2 and $(M+1)$. In the remained state i.e., OFF state, reliable connection can not be achieved because the instantaneous transmission rate exceeds the instantaneous channel capacities, so the service rates in this state is zero.

The normalized effective capacity (in bits $/ \mathrm{s} / \mathrm{Hz}$ or $1 /$ nat) under the average interference power constraint Equation (9) can be formulated as the following optimization problem

$$
E_{c}=-\frac{1}{\theta T B_{c}} \max _{r_{b}, r_{d}>0, S_{b}, S_{d}>0} \log _{e}\left(p_{1} \mathbb{E}\left[\mathrm{e}^{-(T-\tau) \theta r_{b}}\right]+\sum_{m=1}^{M} p_{m+1} \mathbb{E}_{m}\left[\mathrm{e}^{-(T-\tau) \theta r_{d}}\right]+p_{M+2}\right),
$$

s.t. Equation (9) holds, where $p_{m}$ for $m=1,2, \cdots, M+2$ are the state transition probabilities defined in Section 3. The maximization operator is with respect to the power adaptations $S_{b}$ and $S_{d}$.

Note that the expectation $\mathbb{E}\left[\mathrm{e}^{-(T-\tau) \theta r_{b}}\right]$ in the objective function and $\mathbb{E}\left[S_{b} g_{c p}\right]$ in the constraint of the above optimization problem are with respect to the joint distribution of $\left(g, g_{c p}\right)$ of the channel selected for transmission when all channels are sensed busy. The expectations $\mathbb{E}_{m}\left[\mathrm{e}^{-(T-\tau) \theta r_{d}}\right]$ and $\mathbb{E}_{m}\left[S_{d} g_{c p}\right]$ are with respect to the joint distribution of $\left(g, g_{c p}\right)$ of the channel selected for transmission when $m$ channels are 
sensed as idle.

To identify the optimal power allocation that the cognitive users should employ, the problem in Equation (11) can be converted to a minimization problem using the fact that logarithmic function is a monotonic function (Equation (12)). s.t. Equation (9) holds.

This objective function is strictly convex and the constraint function in Equation (11) is linear with respect to $S_{b}$ and $S_{d}$. This can be concluded from the fact that strict convexity follows from the strict concavity of $r_{b}$ and $r_{d}$ in Equations (4) and (5) with respect to $S_{b}$ and $S_{d}$, strict convexity of the exponential function, and the fact that the weighted sum of strictly convex functions is strictly convex $[8,9]$. Using Lagrangian method, the power allocation can be expressed as

$$
S_{b}^{*}= \begin{cases}\frac{N_{b}}{g}\left(\left(\frac{z}{w_{b}}\right)^{\frac{1}{\alpha+1}}-1\right), \frac{z}{w_{b}} \geq 1 ; \\ 0, & \text { otherwise, }\end{cases}
$$

and

$$
S_{d}^{*}= \begin{cases}\frac{N_{d}}{g}\left(\left(\frac{z}{w_{d}}\right)^{\frac{1}{\alpha+1}}-1\right), & \frac{z}{w_{d}} \geq 1 ; \\ 0, & \text { otherwise, }\end{cases}
$$

where $\alpha=\frac{B_{c}(T-\tau) \theta}{\log 2}, w_{b}=\frac{N_{b} q p_{d}}{\alpha \rho} \lambda$, and

$w_{d}=\frac{N_{d} q\left(1-p_{d}\right)}{\alpha(1-q)\left(1-p_{f}\right)} \lambda$.

Using these optimal transmission powers, the effective capacity can be expressed in integral form as Equation (15).

\section{Selection Criterion in Rayleigh Fading Channel}

We aim present a criterion with which the transmission channel is selected from a set of available channels. Since the terms $\left(z / w_{b}\right)^{\frac{-\alpha}{\alpha+1}}$. and $\left(z / w_{d}\right)^{\frac{-\alpha}{\alpha+1}}$ in Equation (13) are monotonically decreasing functions of $z$. The term:

$\left[-\log _{e}\right.$ (monotonically decreasing functions) $]$ is increasing function. It can be observed from Equation (15) that the effective capacity depends only on the channel power ratio $z$, and it is increasing with increaseing this power ratio. Therefore, the criterion for choosing the transmission band among multiple busy bands unless there is no idle band detected, or among multiple idle bands if there are idle bands detected should be based on this ratio of the channel gains. Clearly, the policy that maximizes the effective capacity is to choose the channel with the highest ratio of $z$. This also intuitively leads to a result that as we want to maximize $g$ to improve the secondary transmission and at the same time minimize $g_{c p}$ to diminish the interference caused to the primary users. Maximizing $z$ provides us the problem key in the channel selection algorithm.

Let $w=\max \left\{\frac{g_{m}}{g_{c p, m}}\right\}, \forall m=1, \cdots, M$, where $\frac{g_{m}}{g_{c p, m}}$ is the ratio of the gains in the $m^{\text {th }}$ band channel. All these ratios are assumed to be independent and identical distributed.

Statistically if $Y=\max \left(X_{1}, \cdots, X_{n}\right)$, where $X_{i}$ are independent and identically distributed random variables with $p d f$ of $f_{x}(x)$ then the $c d f$ and $p d f$ of $Y$ are given as (Theorem 5.7 in [10])

$$
\begin{gathered}
F_{Y}(y)=\left[F_{X}(y)\right]^{n}, \\
f_{Y}(y)=\frac{\mathrm{d} F_{Y}(y)}{\mathrm{d} y}=n f_{X}(y)\left[F_{X}(y)\right]^{n-1} .
\end{gathered}
$$

Using this fact, we can express the $p d f$ of $w$ as

$$
f(w)=M f_{z}(w)\left(F_{z}(w)\right)^{M-1}
$$

where $f_{z}$ and $F_{z}$ are the $p d f$ and $c d f$ of the gain ratio $z$ in one channel, respectively.

Now, the first integral in Equation (15) can be evaluated with respect to this distribution.

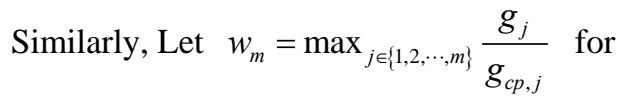
$m=1,2, \cdots, M$. The $p d f$ of $w_{m}$ can be expressed as follows:

$$
f_{m}(w)=m f_{z}(w)\left(F_{z}(w)\right)^{m-1}, m=1,2, \cdots, M
$$

The second integral of Equation (15) can be evaluated

$$
\begin{gathered}
E_{c}=\min _{r_{b}, r_{d}>0, S_{b}, S_{d}>0}\left\{p_{1} \mathbb{E}\left[\mathrm{e}^{-(T-\tau) \theta r_{b}}\right]+\min _{r_{b}, r_{d}>0, S_{b}, S_{d}>0} p_{1} \mathbb{E}\left[\mathrm{e}^{-(T-\tau) \theta r_{b}}\right]+p_{M+2}\right\}, \\
E_{c}=-\frac{1}{\theta T B_{c}} \log _{e}\left[p_{1} \int_{w_{b}}^{\infty}\left(\frac{z}{w_{b}}\right)^{\frac{-\alpha}{\alpha+1}} f(z) \mathrm{d} z+\sum_{m=1}^{M} p_{m+1} \int_{w_{b}}^{\infty}\left(\frac{z}{w_{b}}\right)^{\frac{-\alpha}{\alpha+1}} f_{m}(z) \mathrm{d} z+p_{M+2}\right]
\end{gathered}
$$


using this distribution, and by using the power series property [3]:

$$
\sum_{m=1}^{M} \frac{m M !}{(M-m) !} \rho^{M-m}(1-\rho)^{m-1} x^{m-1}=M((1-\rho) x+\rho)^{M-1}
$$

The analysis in the preceding sections applies for arbitrary joint distributions of $g$ and $g_{c p}$. Channel fading is assumed here to be Rayleigh fading in which the power gains $g$ and $g_{c p}$ are exponentially distributed. We further assume that these fading are mutually independent and each has unit-mean. Statistically, if there are two independent exponentially distributed variables $X$ and $Y$, the random variable $Z=\frac{X}{Y}$ has the $p d f$

$$
f_{z}(z)=\frac{1}{(z+1)^{2}}, z \geq 0 .
$$

So, the $p d f$ of $w$ in Equations (18) and (19) can be expressed as

$$
\begin{aligned}
& f(w)=\frac{M}{w^{2}}\left(\frac{w}{w+1}\right)^{M+1} \\
& f_{m}(w)=\frac{m}{w^{2}}\left(\frac{w}{w+1}\right)^{m+1}
\end{aligned}
$$

Using Equations (6), (7), (15), (21), the effective capacity formula for Rayleigh fading channel can be written as in (22), where

$\alpha_{1}=\alpha /(\alpha+1), \alpha_{2}=(2 \alpha+1) /(\alpha+1)$. The impact of several parameters on the effective capacity is investigated through the following numerical example (Equation (22)).

\section{Numerical Example}

In this section, several numerical results for the obtained effective capacity expression will be investigated. The impact of channel sensing parameters and the average interference constraints are illustrated. The frame duration is $T=1 \mathrm{~s}$ with $10 \%$ of this time is conserved for sensing process. The prior probability of the channel being busy is the same for all channels and set to $q=0.1$. All variances are set to unity. The channel bandwidth is $B=10 \mathrm{KHz}$.

Figure 2, shows the effective capacity vs detection probability $p_{d}$, for different number of channels $M$ when the interference constraint is normalized by the noise power. As $p_{d}$ increases, the effective capacity increases due to the fact that more reliable detection of the activity primary users leads to fewer miss-detection. It can also be seen that the highest effective capacity is attained when $M=1$. Hence, the cognitive users does not benefit from the availability of multiple channels. This is especially pronounced for high values of $p_{d}$. Although several parameters affect the value of the effective capacity, one explanation for this observation is that the probabilities of the cases $C_{b b}$ and $C_{b d}$, in which the cognitive users transmit with power $S_{b}$, decrease with increasing $M$, while the probabilities of the cases $C_{d b}$ and $C_{d d}$ in which the cognitive users transmit with $p$ ower $S_{d}$, increase.

In Figure 3, the effect of the primary user's occupancy $q$ on the effective capacity is investigated for different number of sensed channels. This effect is observable for relatively small detection probability. While for higher ( i.e. , $p_{d}>0.8$ ), the improvement in the effective capacity decreases. This is most likely true for all $M>1$. For $M=1$, higher $q$ attached with higher $p_{d}$ indicate to most likely of the presence of the primary user and hence less capacity can be achieved by the cognitive user as can be seen from the figure.

Figure 4 examines the impact of the $Q o S$ exponent values, $\theta$ for $M=5$. This figure confirms that significant capacity gains can be achieved for lossy $Q o S$ constraints (i.e., small $\theta$ ). While the capacity decreases dramatically for highly strength $Q o S$ constraints.

In Figure 5, the effective capacity is plotted versus the interference threshold $I^{\text {th }}$ for different number of channels. The probability of detection is sit at $p_{d}=0.8$. The figure shows that as the interference gets more strict (i.e., $I^{\text {th }}$ becomes smaller), a higher number of channels is needed to maximize the effective capacity.

As an example, if the interference threshold $I^{\text {th }}<-20 d$, then five channels are needed to reach maximum capacity, while if the interference threshold $I^{\text {th }}<0 \mathrm{~dB}$, one channel is enough to get the highest throughput. As it is mentioned before, increasing the number of available channels from which the transmission channel is selected provides no benefit or can even degrade the performance of the cognitive users. On the other hand, it always provides benefits to the primary users in the sense of lowering the probability of interference.

$$
\begin{aligned}
E_{c}=-\frac{1}{\theta T B_{c}} \log _{e} & {\left[M \rho^{M}\left(w_{b}\right)^{\alpha_{1}} \int_{w_{b}}^{\infty} w^{-\alpha_{2}+M}\left(\frac{1}{w+1}\right)^{M+1} \mathrm{~d} w+(1-q)\left(1-p_{f}\right)\left(w_{d}\right)^{\alpha_{1}} \sum_{m=1}^{M} m C(M, m) \rho^{M-m}\right.} \\
& \left.+(1-\rho)^{m-1} \int_{w_{d}}^{\infty} w^{-\alpha_{2}+m}\left(\frac{1}{w+1}\right)^{m+1} \mathrm{~d} w+p_{M+2}\right]
\end{aligned}
$$




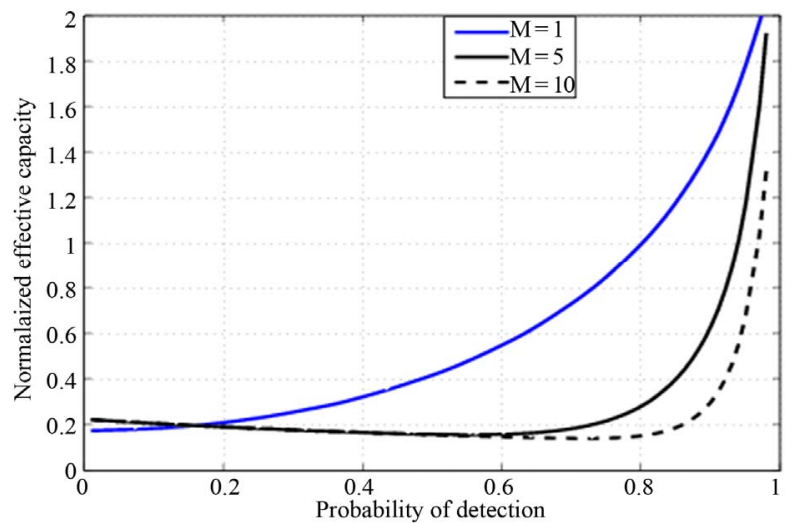

Figure 2. Effective capacity vs probability of detection for different number of channels in the Rayleigh fading channel.

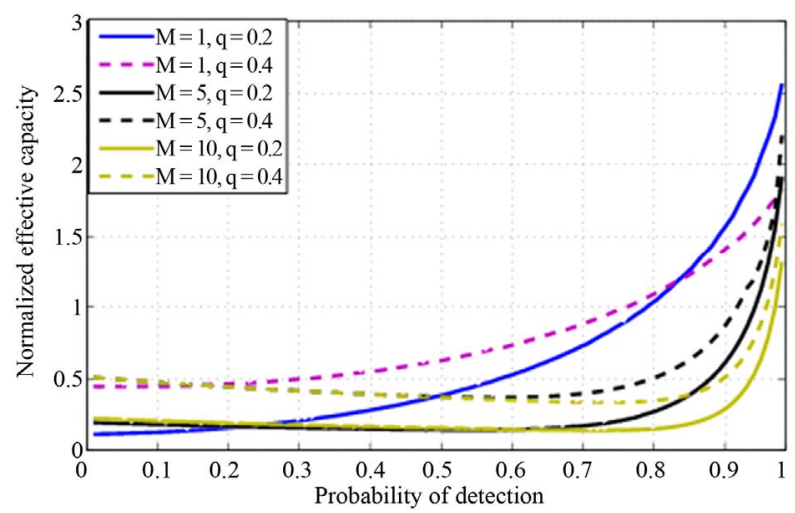

Figure 3. Normalized effective capacity vs probability of detection for different number of channels and different primary probabilities $q, \theta=0.1$. Dashed curves represent for the $q=0.4$ case and solid curves represent the $q=0.2$ case relevant colors.

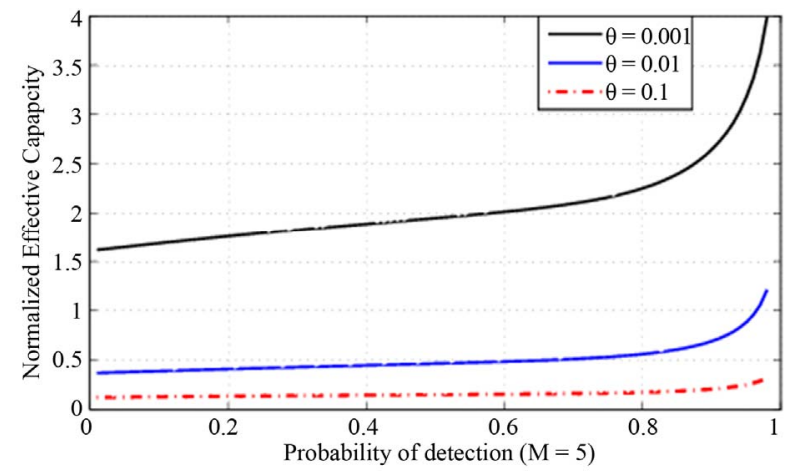

Figure 4. Effective capacity vs probability of detection for different $Q o S$ exponent delay $\theta, M=5, q=0.2$.

\section{Conclusion}

The performance of cognitive transmission under $Q o S$ and interference constraints is studied. Cognitive users

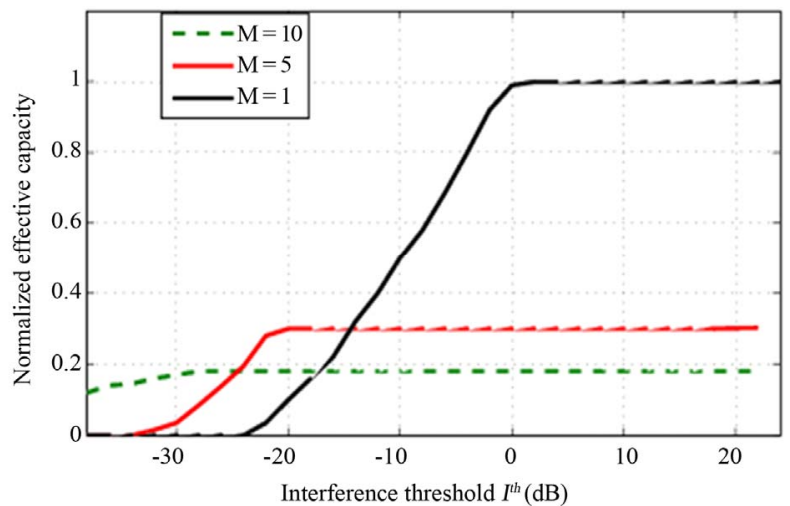

Figure 5. Effective capacity vs $I^{\text {th }}$ for different number of channels in the Rayleigh fading channel, $P_{d}=0.8$ $q=0.2, \theta=0.1$.

are assumed to perform sensing in multiple channels and then select a single channel for transmission with rate and power that depend on both sensing outcomes and fading distribution. A state transition model for this cognitive operation has been constructed. All possible cases and states are considered and analyzed. Interference constraint is statistically analyzed and formulated. Maximum throughput formulas for the cognitive users are obtained. Selection criterion that maximizes the capacity is proposed for arbitrary channel fading then it is applied for Rayleigh distribution. The optimal power allocation is also determined. Sensing multiple bands brings advantages only under strict interference requirements and it reduces the probability of a primary user to be interfered.

\section{REFERENCES}

[1] L. Musavian and S. Aissa, "Quality-of-Service Based Power Allocation in Spectrum-Sharing Channels," IEEE Global Communication Conference GOLBCOM, New Orleans, 30 November-4 December 2008.

[2] L. Musavian and S. Aissa, "Adaptive Modulation in Spectrum-Sharing Systems with Delay Constraints,” IEEE International Conference on Communications ICC, Dresden, 14-18 June 2009.

[3] W. Magnus, F. Oberhettinger and F. Tricomi, "Higher Transcendental Functions,” McGraw Hill, New York, 1982.

[4] Wolfram Mathematica, the Web’s Most Extensive Mathematics Recourses. http://mathworld.wolfram.com

[5] D. Wu and R. Negi, "Effective Capacity: A Wireless Link Model for Support of Quality of Service,” IEEE Transactions on Wireless Communication, Vol. 2, No. 4, 2003, pp. 630-643.

[6] E. Jorswieck, R. Mochaourab and M. Mittelbach, "Effective Capacity Maximization in Multi-Antenna Channels with Covariance Feedback," IEEE Transactions on Wireless Communication, Vol. 9, No. 10, 2010, pp. 2988- 
2993. doi:10.1109/TWC.2010.080210.091060

[7] L. Musavian, S. Aïssa and S. Lambotharan, "Effective Capacity for Interference and Delay Constrained Cognitive Radio Relay Channels," IEEE Transactions on Wireless Communication, Vol. 9, No. 5, 2010, pp. 1698-1707. doi:10.1109/TCOMM.2010.05.090600

[8] S. Boyd and L. Vandenberghe, "Convex Optimization," 7th Edition, Cambridge University Press, Cambridge,
2009.

[9] D. Bertsekas, "Convex Analysis and Optimization,” Athena Scientific, Belmont, 2003.

[10] R. Yates and D. Goodman, "Probability and Stochastic Processes: A Friendly Introduction for Electrical and Computer Engineers,” 2nd Edition, John Wiley \& Sons, Hoboken, 2005, pp. 257-264. 